UMN-TH-1024/1992

MAY 1992

\title{
Stringy Cosmic Strings and Axion Cohomology
}

\author{
Nemanja Kaloper * \\ School of Physics and Astronomy \\ University of Minnesota \\ Minneapolis MN 55455
}

\begin{abstract}
The static stationary axially symmetric background ("infinite cosmic string") of the $D=4$ string theory provided with an axion charge is studied in the effective theory approach. The most general exact solution is constructed. It is found that the Kalb-Ramond axion charge, present in the string topology $R^{3} \times S^{1}$, produces nontrivial gravitational field configurations which feature horizons. The corresponding "no-hair" theorems are proved which stress uniqueness of black strings. Connection of the solutions with the gauged WZWN sigma model constructions on the world sheet is discussed since they are the only target spaces which hide their singularities behind horizons, and thus obey the cosmic censorship conjecture.
\end{abstract}

* Address after Sep. 1, 1992: Dept. of Physics, Univ. of Alberta, Edmonton, Alta T6G 2J1, Canada 


\section{Introduction}

Interest in cosmic strings as possible gravitational solutions has arisen in response to the study of cosmological phase transitions in the early Universe. Investigations of mechanisms of the phase transitions have led us to believe that the field configurations specific to theories with spontaneously broken symmetries can provide the necessary energy-momentum sources to support string-like solutions of the coupled gravity-matter equations $^{1)}$. Two generic types of solutions were found, depending on the type of the symmetry which gets broken. When the spontaneously broken symmetry is a local gauge symmetry, the string solution associated with it appears to be devoid of all long-range interactions, due to the low energy cutoffs introduced by the symmetry breaking ${ }^{1,2}$ ) (except, possibly, in case of the superconducting cosmic string, which may have electromagnetic long-range interactions with interstellar plasma $\left.{ }^{3)}\right)$. Thus, the only non-trivial gravitational effect produced by a local string is the deficit angle, a phenomenon truly topological in its nature.

The situation is significantly different in case of global strings. These were shown to be consistent gravity-matter configurations in theories with broken global symmetries. Long-range gravitational interactions persist in this case due to the coupling of gravity to Goldstone bosons left after the symmetry breaking. Presence of the Goldstone bosons actually assures there are no low energy cutoffs that rule out long-range interactions for local strings. However, for the very same reason the gravitational field associated with global strings is not asymptotically flat, since the energy-momentum density of the matter does not fall off to zero rapidly enough. The gravitational force of the string, curiously,

turns out to be repulsive ${ }^{4-6)}$. A peculiarity of presence of a horizon in the geometry of a global string was observed by Harari and Polychronakos ${ }^{7)}$ in the limit of small Goldstone boson energy density (necessary in their analysis for establishing boundary conditions at 
the string core), and further elucidated by Gibbons et $\mathrm{al}^{8)}$. In particular, the analysis of Ref. [ 8 ] points out general circumstances under which global cosmic strings exist, which are singular and possess event horizons. It is interesting to note that in order to find such solutions, Gibbons et al. resort to string theoretic sigma model target spaces as matter sources.

Stringy aspects of cosmic strings have already been investigated ${ }^{9-11)}$, along with cosmic strings in other nonminimal theories of gravity ${ }^{12)}$. A common feature of such treatises is that all these theories are characterized with the presence of an extra scalar field non-minimally coupled to gravity and/or matter, so that its variation produces non-trivial gravitational field exterior to a string even for local cosmic strings. Of particlular interest here are the recent studies which attempt to extend the $1+1$ Witten black hole solution ${ }^{13)}$ and its conformal field theory interpretation to higher dimensions. Some solutions of the gauged Wess-Zumino-Witten-Novikov (WZWN) sigma models on axially symmetric static target spaces have been constructed already ${ }^{14-18)}$. More comprehensive approaches are under way ${ }^{19)}$. Also, investigations of topological configurations in the massless sector of the theory have been conducted, leading to supersymmetric instanton solutions ${ }^{20)}$.

In this paper a class of exact solutions with an axion charge will be derived and analyzed. It will be demonstrated that classically they represent global string-like solutions with different analytic properties due to the presence of a nontrivial dilaton field and axion charge around it. A black string version of the "no-hair" theorem will be proved, showing uniqueness of a class of charged $4 D$ black strings with horizons. Those are singled out, as they correspond to the WZWN models and represent exact conformal field theories on the string worldsheet. As such, they are viable candidates for the string field theory vacua. 


\section{Axion Cohomology}

The starting point of our investigation is the action for the bosonic sector of the supergravity multiplet in the background field formulation of string theory. In the Einstein frame (in all that follows Yang-Mills fields are ignored) to order $O\left(\alpha^{0}\right)$ it is

$$
S=\int d^{4} x \sqrt{g}\left(\frac{1}{2 \kappa^{2}} R-e^{-2 \sqrt{2} \kappa \Phi} H_{\mu \nu \lambda} H^{\mu \nu \lambda}-\frac{1}{2} \partial_{\mu} \Phi \partial^{\mu} \Phi\right)
$$

where $R$ is the Ricci scalar, $H_{\mu \nu \lambda}$ the Kalb-Ramond axion field strength and $\Phi$ is the dilaton. Conventions of Ref. [ 22 ] are followed throughout the paper. Here it was implicitly assumed that the cosmological constant at the string world-sheet level, leading to the exponential dilaton potential in the Einstein frame action, is zero. This assumption can only be justified by inspecting evolution of the structure of vacuum with the complete description of string theory. In the absence of such a description, it is reasonable to imagine that the various conformal anomalies conspire to yield cancellation of the total central charge (for example, the string vacuum could be decomposed as $M^{4} \times K^{6}$ where the internal degrees of freedom yield the cancellation of the central charge, but at low enough energies are dynamically decoupled). Furthermore, the compactification scale is expected to be high above the supersymmetry breaking scale where the dilaton obtains mass. Hence solutions of the theory above may be expected to describe cosmic strings in an early phase of the Universe, somewhere between the compactification and the supersymmetry breaking scales. For the sake of simplicity, we will first ignore the dilaton field and look only at the coupled axion-gravity system. It is our goal here to examine the influence of the nonvanishing axion charge on the gravitational field.

The metric of a cosmic string is described by the cyllindrically symmetric static stationary background, 


$$
d s^{2}=-e^{2 \nu} d t^{2}+e^{2 \lambda} d r^{2}+e^{2 \mu} d z^{2}+e^{2 \eta} d \phi^{2}
$$

where the metric functions depend only on the radial coordinate $r$. The four metric functions are not independent, however, since by a coordinate transformation (for example, $\left.\rho=e^{\eta(r)}\right)$ any combination of them can be fixed. It is convenient to keep all four arbitrary for calculational purposes and simplify the equations of motion by a specific choice. This freedom is a remnant of the full $G L(3,1)$ gauge invariance of gravity in $4 D$.

The equations of motion are, in terms of the dual of the Kalb-Ramond field strength,

$$
V_{\mu}=\sqrt{g} \epsilon_{\nu \lambda \sigma \mu} H^{\nu \lambda \sigma}
$$

of the form (in $\kappa^{2}=1$ units)

$$
R_{\mu \nu}-\frac{1}{2} g_{\mu \nu} R=\frac{1}{3} V_{\mu} V_{\nu}-\frac{1}{6} g_{\mu \nu} V^{2}
$$

and

$$
d V=d^{*} V=0
$$

where $V=V_{\mu} d x^{\mu}$ is the associated 1-form.

Usually, one would search for

$$
V=d a
$$

as the solution of the two axion equations. In the most general case when the base manifold has nontrivial first cohomology,

$$
V=d a+C_{A} \gamma^{A}
$$


where $\gamma^{A}$ are the harmonic forms generating the first cohomology group of the manifold and $C_{A}$ are constants. Upon substitution of the axion solution in the Einstein's equations for the assumed background describing a base manifold of topology $R^{3} \times S^{1}$ one sees that the axion can be represented by a purely topological contribution ${ }^{3)}$

$$
V=Q d \phi
$$

where $Q$ is the axionic charge per unit length of the string:

$$
Q=\frac{1}{2 \pi} \int_{S^{1}} V
$$

This charge generalizes the uniform line distribution of electric charge, as is obvious from $Q \sim \int_{S^{1}} * H$

Clearly, due to its topological nature expressed via the Gauss law above, the charge $Q$ is conserved. Furthermore, we can rewrite the energy-momentum tensor of this configuration as

$$
T_{\mu \nu}=\frac{Q^{2}}{6} e^{-2 \eta} \operatorname{diag}(1,-1,-1,1)
$$

recognizing it as the matter source giving rise to the gravitational field of a global cosmic string. This should not be a surprise, since Gibbons et al. ${ }^{8)}$ showed that global strings arise whenever matter sources can be identified with target spaces containing closed geodesics. As a consequence, gravitational field of global strings arises when the principal bundle describing matter content is of nontrivial first cohomology. In the case demonstrated above where $V=Q d \phi$ one can identify the space-time angle $\phi$ as a representation of a matter field living on a $U(1)$ target manifold, a closed target space geodesic by itself. Then ${ }^{1,2,4,5)}$ it is easy to write down the solution for the gravitational field around such a cosmic string: 


$$
\begin{aligned}
d s^{2}= & -\left(\frac{r}{r_{0}}\right)^{1-w} d t^{2}+\left(\frac{r}{r_{0}}\right)^{1+w} d z^{2} \\
& +\Delta^{2}\left(\frac{r}{r_{0}}\right)^{\frac{w^{2}-1}{2}} \exp \left(-\frac{Q^{2}}{6}\left(\frac{r}{r_{0}}\right)^{2}\right) d r^{2}+\Psi^{2}\left(\frac{r}{r_{0}}\right)^{\frac{w^{2}-1}{2}} \exp \left(-\frac{Q^{2}}{6}\left(\frac{r}{r_{0}}\right)^{2}\right) d \phi^{2}
\end{aligned}
$$

Here $w, r_{0}, \Delta$ and $\Psi$ are constants of integration. Observe, that when $Q=0$ the solution above correctly reduces to the static cyllindrically symmetric metric solving the vacuum Einstein's equations, i.e., to the class of solutions containing the local cosmic string (to demonstrate this one would have to take the zero limit of other parameters in (11) as well). With the help of a coordinate transformation, the case $w=-1$ can be cast in the form first discussed by Harari and Polychronakos ${ }^{4)}$,

$$
\begin{aligned}
d s^{2}= & -\left(1-\frac{1}{\sigma} \ln \left(\frac{\rho}{\rho_{0}}\right)\right) d \tau^{2}+d z^{2} \\
& +\left(1-\frac{1}{\sigma} \ln \left(\frac{\rho}{\rho_{0}}\right)\right)^{-1} d \rho^{2}+\frac{Q^{2} \sigma}{3} \rho^{2} d \phi^{2}
\end{aligned}
$$

where $\sigma=\ln \Delta$ and $\rho_{0}^{2}=\frac{3 r_{0}^{2}}{Q^{2} \sigma}$. The Kalb-Ramond axion then is

$$
H=\frac{1}{2 Q \sigma \rho^{2}} d \tau \wedge d z \wedge d \rho
$$

Note that in the limit $Q \rightarrow 0, \sigma \rightarrow \infty, Q^{2} \sigma=$ const. equations (12-13) reduce to the local cosmic string, as claimed above.

The difference between this solution and the one previously analyzed by Harari and Polychronakos is that it is an exact, everywhere valid solution representing a doubly singular line distribution of axial charge, the singularities being located at the origin and at infinity. Note, however, the strange property of the above solution, that if we allow imaginary axion charge, and at the same time flip the sign of $\sigma$, we obtain the (more appealing) configuration where the physical region of the metric (12) is outside of the horizon $\rho_{h}=\rho_{0} \exp \sigma$, as opposed to the solution of Harari and Polychronakos, who find a rather peculiar situation where the physical region of the metric is inside it. In this case, the metric (12) has a well defined Newtonian limit, the gravitational potential being 


$$
\Phi_{N}=-\frac{1}{2|\sigma|} \ln \frac{\rho}{\rho_{0}}
$$

exactly the expression for the Newtonian potential of a nonrelativistic line distribution of mass with density per unit length $2 \pi|\sigma|^{-1}$. Then it is easy to understand the appearance of the singularity at infinity. This is just the usual infrared divergence in the classical limit of a theory describing massless particles.

It is interesting to note that the need for an imaginary axion to produce the solution (12) with $\sigma>0$ can be relaxed if the three form $H_{\mu \nu \lambda}$ has been given a tachyonic kinetic term instead. Such a degree of freedom might arise, for example, after dimensional reduction of a higher rank form defined in some background metric with compactified internal space of pseudoeuclidean signature.

\section{The Supergravity Multiplet}

We will now investigate the cylindrically symmetric static solutions with axionic charge on the target manifold of topology $R^{3} \times S^{1}$ in the theory describing the complete bosonic sector of the supergravity multiplet. It contains the graviton, the dilaton and the three form Kalb-Ramond axion. This time, however, we choose to work in the world sheet frame, which has been argued to represent the more natural background for discussing the string propagation in curved backgrounds, its role being exactly the string coupling constants. Also, this will be more convenient for later comparison with the WZWN constructions. Of course, the analysis of the background field equations of motion, understood as a classical theory, really does not depend on which frame one chooses to work in, since a simple conformal transformation relates the equations of motion in different frames. 
Thus, the action describing the theory on the target space is, to order $O\left(\alpha^{0}\right)$ in $4 D$,

$$
S=\int d^{4} x \sqrt{G} e^{-\sqrt{2} \kappa \Phi}\left(\frac{1}{2 \kappa^{2}} R-H_{\mu \nu \lambda} H^{\mu \nu \lambda}+\partial_{\mu} \Phi \partial^{\mu} \Phi+\Lambda\right)
$$

where the cosmological constant has been included for the background theory above to represent the noncritical string theory too. The world sheet cosmological constant can arise from the leftover conformal anomaly which may not have been cancelled between the central charges of the particle and ghost systems. Then, the requirement of cylindrical symmetry of the target space is equivalent to using the metric (2) on the world sheet and allowing the dilaton to depend on the radial coordinate only. As for the axion, since we are interested in picking it so that it is given by the $S^{1}$ cohomology of the manifold, its equations of motion $d H=0$ and $d \exp (-\sqrt{2} \kappa \Phi)^{*} H=0$ in conjunction with the duality transformation and the metric ansätz, yield

$$
\begin{aligned}
B & =2 B_{20} d z \wedge d t \\
H & =2 B_{20}^{\prime} d r \wedge d z \wedge d t \\
B_{20}^{\prime} & =-\frac{Q}{2} \exp (\sqrt{2} \kappa \Phi+\nu+\mu+\lambda-\eta)
\end{aligned}
$$

where the prime denotes a derivative with respect to $r$. The constant of integration $Q$ represents the axion cohomology charge, defined via the Gauss law (9), which in this case can be writen as

$$
Q=\frac{1}{2 \pi} \int_{S^{1}} e^{-\sqrt{2} \kappa \Phi *} H
$$

It is easiest to work in the action on the tangent bundle to derive the equations of motion. Defining the locally flat coordinate system with tetrads $e^{\alpha}=\exp \left(\nu_{\alpha}\right) d x^{\alpha}$ (no summation) with $\alpha \in\{0, \ldots, 3\}$ and computing the connexion and curvature forms, the action can be rewritten in terms of the degrees of freedom, in units of Planck's mass $\left(\kappa^{2}=1\right)$, as 


$$
\begin{aligned}
S=\int d r\{ & \left(\nu^{\prime} \mu^{\prime}+\nu^{\prime} \eta^{\prime}+\mu^{\prime} \eta^{\prime}-\sqrt{2} \Phi^{\prime}\left(\nu^{\prime}+\mu^{\prime}+\eta^{\prime}\right)\right) e^{\nu+\mu+\eta-\lambda-\sqrt{2} \Phi} \\
& +\frac{\Upsilon^{2}}{6} e^{\eta-\nu-\mu-\lambda-\sqrt{2} \Phi} \\
& \left.+\Lambda e^{\nu+\mu+\eta+\lambda-\sqrt{2} \Phi}+\Phi^{\prime 2} e^{\nu+\mu+\eta-\lambda-\sqrt{2} \Phi}\right\}
\end{aligned}
$$

where $\Upsilon=Q \exp (\nu+\mu+\lambda-\eta+\sqrt{2} \Phi)$ is obtained after substitution of the axion solution (16).

Variation of the action (18) gives the equations of motion. With some simple algebra, and after the choice of gauge $\lambda=\nu+\mu-\sqrt{2} \Phi$ has been made, they become:

$$
\begin{gathered}
\eta^{\prime \prime}+\eta^{2}=0 \\
\mu^{\prime \prime}-\nu^{\prime \prime}+\left(\mu^{\prime}-\nu^{\prime}\right) \eta^{\prime}=0 \\
\mu^{\prime \prime}+\nu^{\prime \prime}+\left(\mu^{\prime}+\nu^{\prime}\right) \eta^{\prime}=\frac{2}{3} Q^{2} e^{2(\nu+\mu-\eta)} \\
\Phi^{\prime \prime}+\Phi^{\prime} \eta^{\prime}=\frac{\sqrt{2}}{3} Q^{2} e^{2(\nu+\mu-\eta)}-\sqrt{2} \Lambda e^{2(\nu+\mu-\sqrt{2} \Phi)} \\
\nu^{\prime} \mu^{\prime}+\nu^{\prime} \eta^{\prime}+\mu^{\prime} \eta^{\prime}-\sqrt{2} \Phi^{\prime}\left(\nu^{\prime}+\mu^{\prime}+\eta^{\prime}\right)+\Phi^{\prime 2}=\Lambda e^{(\nu+\mu-\sqrt{2} \Phi)}+\frac{Q^{2}}{6} e^{(\nu+\mu-\eta)}
\end{gathered}
$$

The system of equations above is exactly integrable. Clearly, the function $\eta$ serves the role of the "evolution kernel" and is determined by a Riccati equation which in this case is easy to solve. There exist two different classes of solutions, determined as follows. The general (inequivalent) solutions of the Riccati equation are $\eta=\eta_{0}$ or $\eta=\eta_{0}+\ln \left(r+r_{0}\right)$ where $\eta_{0}$ and $r_{0}$ are integration constants. The constant $r_{0}$ is irrelevant and may be dropped. The other constant, however, may be physically significant as it measures the deficit angle. The remaining equations can be simplified upon substitution of these formulas and integrated. Hence, the solutions are 
Case 1. :

$$
\begin{gathered}
\eta=\eta_{0} \\
\mu-\nu=Y_{0}+Y_{1} r \\
\mu+\nu=X \\
\sqrt{2} \Phi=X-W \\
X^{\prime}= \pm \sqrt{2 C-Y_{1}^{2}+\frac{2}{3} Q^{2} \exp 2\left(X-\eta_{0}\right)} \\
W^{\prime}= \pm \sqrt{C+2 \Lambda \exp (2 W)}
\end{gathered}
$$

and

Case 2. :

$$
\begin{gathered}
\eta=\eta_{0}+\ln r \\
\mu-\nu=Y_{0}+Y_{1} \ln r \\
\mu+\nu=X \\
\sqrt{2} \Phi=X+\eta-W \\
X^{\prime}= \pm \frac{\sqrt{2(C-1)-Y_{1}^{2}+\frac{2}{3} Q^{2} \exp 2\left(X-\eta_{0}\right)}}{r} \\
W^{\prime}= \pm \frac{\sqrt{C+2 \Lambda \exp 2\left(W-\eta_{0}\right)}}{r}
\end{gathered}
$$

Here $\eta_{0}, Y_{0}, Y_{1}$ and $C$, together with $\nu_{0}$ and $\mu_{0}$ are the (independent) integration constants which completely determine solutions. We will always assume $Q \neq 0$, as we are interested in the effects of the axion cohomology. Besides, the case $Q=0$ is equivalent to the Jordan-Brans-Dicke cosmic string of ref. [ 12 ]. Furthermore, we will require that $\Lambda=-\frac{2}{3} \delta c_{T}=\frac{2}{3}\left(c_{T}-4\right) \geq 0$, as is known to be the case in string theory ${ }^{22)}$. The central charge deficit $\delta c_{T}$ arises via the algebraic construction of the target manifold. The $\lambda$ is determined then with the gauge choice above. 
Typically, the integrals above are combinations of polynomials and hyperbolic and trigonometric functions. The trigonometric functions, however, are highly undesirable, since their periodicity compactifies the radial direction and introduces an infinite number of ring-like singularities inconsistent with the assumed cylindrical structure of the target space. Avoiding them is guaranteed with choice $C=g_{0}^{2}$, and $2 g_{0}^{2}-Y_{1}^{2}=f_{0}^{2}$ or $2 g_{0}^{2}-Y_{1}^{2}-2=f_{0}^{2}$, respectively.

The explicit solutions can be classified with respect to the values of $C, Y_{1}, Q$ and $\Lambda$. They are listed below.

Strings with $\Lambda=0$ :

$$
\begin{gathered}
d s^{2}=-\sqrt{\frac{3}{2}} \frac{f_{0} e^{\eta_{0}}}{Q} \frac{\left(e^{-Y_{1} r} d t^{2}-e^{Y_{1} r} d z^{2}\right)}{\sinh \left(A \mp f_{0} r\right)}+e^{2 W_{0} \pm 2 g_{0} r} d r^{2}+e^{2 \eta_{0}+2 \sigma r} d \phi^{2} \\
e^{-\sqrt{2} \Phi}=\sqrt{\frac{2}{3} \frac{Q e^{-\eta_{0}}}{f_{0}} e^{W_{0} \pm g_{0} r-\sigma\left(\eta_{0}+r\right)} \sinh \left(A \mp f_{0} r\right)} \\
B_{20}=D \pm \frac{3}{4} \frac{f_{0}}{Q} e^{(1+\sigma) \eta_{0}} \operatorname{coth}\left(A \mp f_{0} r\right)
\end{gathered}
$$

Strings with $\Lambda>0$ :

$$
\begin{gathered}
d s^{2}=-\sqrt{\frac{3}{2}} \frac{f_{0} e^{\eta_{0}}}{Q} \frac{\left(e^{-Y_{1} r} d t^{2}-e^{Y_{1} r} d z^{2}\right)}{\sinh \left(A \mp f_{0} r\right)}+\frac{g_{0}^{2}}{2 \Lambda} \frac{d r^{2}}{\sinh ^{2}\left(B \mp g_{0} r\right)}+e^{2 \eta_{0}+2 \sigma r} d \phi^{2} \\
e^{-\sqrt{2} \Phi}=\frac{Q e^{-\eta_{0}} g_{0}}{f_{0} \sqrt{3 \Lambda}} \frac{\sinh \left(A \mp f_{0} r\right)}{\sinh \left(B \mp g_{0} r\right)} e^{-\sigma\left(\eta_{0}+r\right)} \\
B_{20}=D \pm \frac{3}{4} \frac{f_{0}}{Q} e^{(1+\sigma) \eta_{0}} \operatorname{coth}\left(A \mp f_{0} r\right)
\end{gathered}
$$

with $2 g_{0}{ }^{2}=f_{0}{ }^{2}+Y_{1}^{2}+2 \sigma$. The parameter $\sigma$ can take values 0 and 1 . A coordinate transformation has been performed to bring the solution of the second case to the form above. Note that in case of $\Lambda$ of opposite sign the only change would be replacing $\sinh \left(B \mp g_{0} r\right)$ with $\cosh \left(B \mp g_{0} r\right)$.

The coordinate frame chosen for representing the solutions above is very transparent for further analysis. We note a few interesting features of these configurations. It is not 
difficult to evaluate the curvature invariants of (22-23) and inspect the type of potential singular behaviour in the metric (we absorb \pm in the parameters).

$$
\begin{aligned}
R=-2 e^{-2 W_{0}+2 g_{0} r} & \left(\frac{7}{4} \frac{f_{0}^{2}}{\sinh ^{2}\left(A+f_{0} r\right)}-f_{0} g_{0} \operatorname{coth}\left(A+f_{0} r\right)+\frac{f_{0}^{2}+g_{0}^{2}}{2}\right. \\
& \left.+\sigma\left(f_{0} \operatorname{coth}\left(A+f_{0} r\right)+g_{0}\right)+\sigma^{2}-\frac{\sigma}{2}\right)
\end{aligned}
$$

and

$$
\begin{gathered}
R=-\frac{4 \Lambda}{g_{0}^{2}} \sinh ^{2}\left(B+g_{0} r\right)\left(\frac{7}{4} \frac{f_{0}^{2}}{\sinh ^{2}\left(A+f_{0} r\right)}-f_{0} g_{0} \operatorname{coth}\left(A+f_{0} r\right) \operatorname{coth}\left(B+g_{0} r\right)\right. \\
\left.+\frac{f_{0}^{2}+g_{0}^{2}}{2}+\sigma\left(g_{0} \operatorname{coth}\left(B+g_{0} r\right)-f_{0} \operatorname{coth}\left(A+f_{0} r\right)\right)+\sigma^{2}-\frac{\sigma}{2}\right)
\end{gathered}
$$

We will first concentrate on the noncritical string case. Assume $g_{0} A \neq f_{0} B$. Then, the solutions contain a singularity determined by $r_{s}=-\frac{A}{f_{0}}$. Hence they are charts of the target valid only on open intervals $r \in\left(-\infty, r_{s}\right)$ and $r \in\left(r_{s}, \infty\right)$. On the other hand, the surface $r_{\infty}=-\frac{B}{g_{0}}$ is well behaved, although the coefficient of $d r^{2}$ diverges here. The curvature actually vanishes here. Inspection of (25) leads to the conclusion that in order to prevent blowing up of the curvature at "spatial infinities", one has to require $f_{0}-g_{0}= \pm \sigma$ (with the "regular" point at $\pm \infty$ ). Note that this is just a necessary but not sufficient condition for regularity. That is the reason for the quotation marks. Regularity at the point would still have to be doublechecked. Only when $\sigma=0$ will both "spatial infinities" be nonsingular. Regularity of one implies regularity of the other. When $\sigma=1$, there appears another singularity at one of the "infinities".

The constraint $2 g_{0}^{2}=f_{0}^{2}+Y_{1}^{2}+2 \sigma$ translates into $f_{0}^{2} \mp 4 \sigma f_{0}=Y_{1}^{2}$. When $\sigma=0$ it is indeed very stringent: it asserts that $f_{0}=g_{0}= \pm Y_{1}$. This fixes the solution uniquely. It is constructed as follows; for simplicity, assume that all the parameters are positive, and that $f_{0} B<g_{0} A$. This does not restrict generality of the construction. Take the patch $\left(r_{\infty}, \infty\right)$ to describe the exterior of the black string; identify $r_{\infty}$ with the physical spatial 
infinity, and $\infty$ with the (outer) horizon. Note that the choice of $r_{\infty}$ as the physical spatial infinity is motivated by the vanishing curvature there. It tells that such space-time is asymptotically flat. The identification of $\infty$ as the (outer) horizon is consistent as the curvature is finite there, although the metric appears singular. This signals the horizon. Also note that the singularity at $r_{s}$ is pushed "outside" of the space-time, and that asymptotic flatness guarantees that it is completely decoupled from the physical region of the space-time. Similarly, construct the interior from the patch $\left(-\infty, r_{s}\right)$ : flip the roles of the $t$ and $z$ coordinates, identify $-\infty$ with the inner horizon, and note that then $r_{s}$ represents a singularity in the space-time, which is unavoidable because the point $r_{\infty}$ was imbedded in the outside. Finally, interpolate the region between the horizons with a Bianchi I type universe, equivalent to the region of imaginary $r$ and the parameters $g_{0}, f_{0}, Y_{1}$ in the original coordinates. This patchwork can be represented compactly with a transformation of coordinates, and the analytic continuation spelled out above. The transformation is $\rho=g_{0} \operatorname{coth}\left(B+g_{0} r\right)$, with $f_{0}=g_{0}=Y_{1}$, and the solution becomes, with the help of additional simple coordinate transformations,

$$
\begin{gathered}
d s^{2}=-\left(1-\frac{x_{+}}{x}\right) d t^{2}+\left(1-\frac{x_{-}}{x}\right) d z^{2}+\frac{d x^{2}}{2 \Lambda\left(x-x_{+}\right)\left(x-x_{-}\right)}+e^{2 \eta_{0}} d \phi^{2} \\
e^{-\sqrt{2} \Phi}=\frac{Q e^{-\eta_{0}}}{\sqrt{3 \Lambda}} \frac{x}{\sqrt{x_{+} x_{-}}} \\
B_{20}=D^{\prime}-\frac{\sqrt{6}}{4} \frac{\sqrt{x_{+} x_{-}}}{x}
\end{gathered}
$$

where $x_{ \pm}$denote locations of the horizons, and $x \in(0, \infty)$.

This is exactly the gauged WZWN sigma model solution first constructed in $4 \mathrm{D}$ by Raiten $^{16)}$ (with a slight modification, the one-point compactification of the $\phi$ space). The limit when $f_{0} \rightarrow 0$ corresponds to the critical case of the above solution.

In all other cases, when at least one of the "spatial infinities" is a singularity, the situation is dramatically different. For example, suppose again that the parameters are positive, that $f_{0} B<g_{0} A$ and that $-\infty$ is a singularity. The patch $\left(-\infty, r_{s}\right)$ then 
can not be extended continuously by gluing extra pieces to it since both end points are singularities. Assuming that the manifold is connected establishes this as an entire spacetime which contains two naked singularities, one at the origin and the other at infinity. The other patch, $\left(r_{s}, \infty\right)$, contains the point $r_{\infty}$. If $\infty$ were not a singularity $(\sigma=1)$, it could in principle be made singularity-free with the point $r_{\infty}$ identified with the physical spatial infinity, and $\infty$ with a horizon (or the coordinate origin). However, closer scrutiny reveals that unless $f_{0} \in\{0,1\}, \infty$ is a singularity, since the Riemann curvature squared diverges otherwise $\left(\lim _{r \rightarrow \infty} R_{\mu \nu \lambda \sigma} R^{\mu \nu \lambda \sigma} \propto f_{0}\left(f_{0}-1\right) \sinh ^{4}\left(B+f_{0} r\right)\right)$. The solution contains a naked singularity at the origin, and can not be extended past it. For the remaining two values for $f_{0}$, the constraints $2 g_{0}{ }^{2}=f_{0}{ }^{2}+Y_{1}^{2}+2$ and $g_{0}+1=f_{0}$ rule out $f_{0}=1$. Thus, $f_{0}=Y_{1}=0$ and $g_{0}{ }^{2}=1$; the solution in this case is

$$
\begin{gathered}
d s^{2}=-\sqrt{\frac{3}{2}} \frac{e^{\eta_{0}}}{Q} \frac{d t^{2}-d z^{2}}{A+r}+\frac{d r^{2}}{2 \Lambda \sinh ^{2}(B+r)}+e^{2 \eta_{0}+2 r} d \phi^{2} \\
e^{-\sqrt{2} \Phi}=\frac{Q e^{-\eta_{0}}}{\sqrt{3 \Lambda}} \frac{(A+r)}{\sinh (B+r)} e^{-\left(\eta_{0}+r\right)} \\
B_{20}=D-\frac{3}{4} \frac{e^{2 \eta_{0}}}{Q} \frac{1}{A+r}
\end{gathered}
$$

The distinct points of this metric are $r=-A, \pm \infty,-B$. Investigation of the Ricci curvature (25) for this metric then shows that $r_{s}=-A$ and both $\pm \infty$ are singularities. So, any one of the patches $\left(-\infty, r_{s}\right),\left(r_{s}, r_{\infty}\right)$ or $\left(r_{\infty}, \infty\right)$ contains at least one singularity. The fact that the signature of the metric does not change as $r$ passes through $r_{\infty}$ says again that $r_{\infty}$ can at best be identified with the physical spatial infinity but is not a horizon. More properly, a timelike Killing vector transported along a geodesic passing through $r_{\infty}$ does not flip into spacelike. Thus, the solution (27) always describes space-times with naked singularities.

The solutions where either both "spatial infinities" are singular, or with the point $r_{\infty}$ sandwiched between $r_{s}$ and the singular "spatial infinity" are all hopeless, since by necessity they contain naked singularities. 
When $g_{0} A=f_{0} B\left(=0\right.$, by a shift of $r$ ), the singularity $r_{s}$ disappears. Instead, $r_{\infty^{ \pm}}=0^{ \pm}$are regular points. If $\pm \infty$ are also required to be regular, $f_{0}-g_{0}= \pm \sigma$. When $\sigma=0$, the solution is fixed uniquely, with both "spatial infinities" regular. Hence both patches $\left(-\infty, 0^{-}\right)$and $\left(0^{+}, \infty\right)$ represent singularity free regions. In effect, they are exact replicas of each other as can be clearly seen under the transformation $r \rightarrow$ $-r, t \leftrightarrow z$. Note that the patches have constant Ricci curvature $R=-3 \Lambda$ and dilaton $\exp (-\sqrt{2} \Phi)=|Q| e^{-\eta_{0}} / \sqrt{3 \Lambda}$. Actually, one can compute that, at the tangent bundle, the Riemann curvature tensor for this configuration is constant, and so are all the curvature invariants. This solution is completely singularity-free. It is a 3D anti-de-Sitter space-time crossed with a flat circle. Each patch separately is an extremal black string of (26), as shown in the 3D case by Horne and Horowitz ${ }^{15)}$. Then, to patch up the solutions, one can identify $0^{ \pm}$with the physical spatial infinities, and $\pm \infty$ with the outer and inner horizons and interpolate between them with a Bianchi I universe. This in fact again corresponds to an analytically continued coordinate transformation. So, start with $\rho=g_{0} \operatorname{coth}\left(g_{0} r\right)$ and rewrite the solution as

$$
\begin{gathered}
d s^{2}=-\sqrt{\frac{3}{2}} \frac{e^{\eta_{0}}}{Q}\left(\left(\rho-g_{0}\right) d t^{2}-\left(\rho+g_{0}\right) d z^{2}\right)+\frac{d \rho^{2}}{2 \Lambda\left(\rho-g_{0}\right)\left(\rho+g_{0}\right)}+e^{2 \eta_{0}+2 r} d \phi^{2} \\
e^{-\sqrt{2} \Phi}=\frac{|Q| e^{-\eta_{0}}}{\sqrt{3 \Lambda}} \\
B_{20}=D-\frac{3}{4} \frac{e^{2 \eta_{0}}}{Q} \rho
\end{gathered}
$$

Notice that the solution suggests that the base space consists of two identical images $\rho \rightarrow-\rho, t \leftrightarrow z$ glued together along the boundary $\partial_{\rho=0} M$. This is a rather awkward situation, in that one can imagine an observer moving along a radial geodesic who can enter the replica universe passing through the axis of the string. More can be learned from the study of radial geodesics. The geodesic equations can be easily integrated to yield (discarding the flat direction $\phi$ ) 


$$
\begin{gathered}
t^{\prime}=K e^{-\rho} \\
z^{\prime}=L e^{-\rho} \\
\rho^{\prime 2}=N\left(\rho-g_{0}\right)\left(\rho+g_{0}\right)+\sqrt{\frac{3}{2}} \frac{e^{\eta_{0}}}{|Q|} \Lambda\left(K^{2}-L^{2}\right)\left(\rho-g_{0}\right)\left(\rho+g_{0}\right) e^{-2 \rho}
\end{gathered}
$$

This states that no matter where the observer starts from, he/she must stop moving in the radial direction when $\rho \rightarrow g_{0}$. In other words, it seems that the point $g_{0}$ represents a classical turning point where kinetic energy vanishes and motion ceases. Yet, a simple transformation of coordinates $x^{2}=\rho-g_{0}$ confirms the naive expectation that the passage is possible. Then,

$$
4 x^{\prime 2}=N\left(x^{2}+2 g_{0}\right)+\sqrt{\frac{3}{2}} \frac{e^{\eta_{0}}}{|Q|} \Lambda\left(K^{2}-L^{2}\right)\left(x^{2}+2 g_{0}\right) e^{-2 \rho}
$$

and therefore the observer, very slowly, and never passing $g_{0}$ in the original coordinates, crosses the horizon. It appears that he/she can travel all the way past, squeezing through the origin into the region $\rho<0$ and eventually reenter the copy universe. Similar tour through the black hole interior was investigated by Horne and Horowitz in $3 \mathrm{D}^{15}$ ), with the difference that their observer had only one horizon to cross. That would correspond to our case when $g_{0}=0$.

If $\sigma=1$, then both "spatial infinities" turn out to be singular, unless $g_{0}{ }^{2}=1$ (by the already mentioned asymptotic behaviour of the square of Riemann tensor). If $g_{0}^{2}=1$ $\left(f_{0}=Y_{1}=0\right)$, the metric is the same as (27) with $A=B=0$ :

$$
d s^{2}=-\sqrt{\frac{3}{2}} \frac{e^{-\eta_{0}}}{Q} \frac{d t^{2}-d z^{2}}{r}+\frac{d r^{2}}{2 \Lambda \sinh ^{2} r}+e^{2\left(\eta_{0}+r\right)} d \phi^{2}
$$

where both $\pm \infty$ are again singular. Consequently, the patches $(-\infty, 0)$ and $(0, \infty)$ both contain naked singularities. However, the situation here is more resemblant of the Harari and Polychronakos solution ${ }^{7)}$, with an additional bonus that it now possesses manifest 
boost invariance in the $t, z$ plane. Namely, with a coordinate transformation $\rho=\exp (r)$, the solution can be rewritten as

$$
d s^{2}=-\sqrt{6} \frac{e^{-\eta_{0}}}{Q} \frac{d t^{2}-d z^{2}}{\ln \rho}+\frac{2 d \rho^{2}}{\Lambda\left(\rho^{2}-1\right)^{2}}+e^{2 \eta_{0}} \rho^{2} d \phi^{2}
$$

and here the horizon is located at $\rho=1$. Still, due to the presence of the logarythm in the metric, the solution is singular at both the origin and infinity. The singularity at infinity should not come as a surprise since, as argued in the previous section, the metric above has the (quasi) Newtonian limit and the singularity at $\infty$ can be understood as the standard infrared divergence.

For all other solutions, the "spatial infinities" are singular, as can be seen from investigating the curvature invariants. Hence, they all contain naked singularities.

Therefore, it has been demonstrated that the WZWN sigma model construction of Raiten is the unique axionically charged cyllindrically symmetric target space of the $4 D$ supergravity multiplet in string theory with central charge deficit which does not have naked singularities, i.e. obeys cosmic censorship! The other solution which was found to be free of naked singularities represents an entire space-time without singularities, but with horizons. It corresponds to an extremal black string solution. It can be extended by gluing an identical copy resulting in a total space-time where observer can travel from one region into the other.

In a sense, this result could be dubbed the "no-hair" theorem for black strings, saying that the strings are described by their mass, axion charge and external dilaton hair, and develop well behaved horizons. We will not dwell here on the global properties of this solution as it has already been studied ${ }^{15,16)}$. We remark however that in order to obtain the full picture about the global properties oh the solution, one should study geodesics of conformal transforms of the world sheet metric. They describe motion of particles with different conformal charges (scaling dimensions) and thus couple differently to the 
dilaton $^{23)}$. The solution (28) is thence standing out as motion of probes in it is universal, irrespective of the nature of test particles, due to the constancy of the dilaton.

The string theory targets with no central charge deficit are analyzed in exactly the same way. The task is much easier here, though. Repeating the study of the singularity structure of the solution (22) along the lines outlined above, one can easily verify that none of these solutions contain a point equivalent to $r_{\infty}$, and contain a singularity at the equivalent of $r_{s}$. Furthermore, the "spatial infinities" there are also singular. Thus, every such solution involves naked singularities. So there are no black strings among the string theory target spaces that bear no central charge.

\section{Summary}

Studies of the nonperturbative aspects of string theory have recently produced novel, unorthodox solutions to the effective theory which appear to bear resemblance to the conventional black holes. They involve nontrivial causal structure of the space-time, and feature horizons. Here general solutions of the 4D effective theory for the supergravity multiplet with cylindrically symmetric target spaces have been studied. It was found that the only solutions consistent with the (hoped for) cosmic censorship are indeed the gauged WZWN sigma models. These solutions are just the 3D black hole of Horne and Horowitz, crossed with a circle, which is necessary to carry the axion charge. In $4 \mathrm{D}$ it is given by an integral of a one-form, and the subspace over which the integration is performed must be compact to give rise to a finite charge. They appear to be the unique spacetime configurations which give rise to event horizons and thus in a sense justify the trust

of representing universal theories that may be used to describe the string theory ground states. 
These solutions may also have relevance in cosmology, as it is well known that there is a simple relationship between static axially symmetric geometries and Bianchi I anisotropic universes. In that case, the axion charge could be interpreted as a homogeneous distribution of axion condensate in the universe, irregardles of whether the universe had a compactified direction or not. Some of similar solutions were considered recently by Tseytlin ${ }^{24)}$.

\section{Acknowledgements}

The author would like to thank K. Olive for helpful conversations. This work has been supported in part by the University of Minnesota Doctoral Dissertation Fellowship. 


\section{References}

[1] A. Vilenkin, Phys. Rev. D23 (1981) 852; Phys. Rep. 121 (1985) 263.

[2] D. Garfinkle, Phys. Rev. D32 (1985) 1323.

[3] E. Witten, Relativistic Astrophysics Proceedings, Chicago 1986 (1986) 606.

[4] D. Harari and P. Sikivie, Phys. Rev. D37 (1988) 3438.

[5] A.G. Cohen and D.B. Kaplan, Phys. Lett. B215 (1988) 67.

[6] R. Gregory, Phys. Lett. B215 (1988) 663.

[7] D. Harari and A.P. Polychronakos, Phys. Lett. B240 (1990) 55.

[8] G.W. Gibbons, M.E. Ortiz and F. Ruiz Ruiz, Phys. Lett. B240 (1990) 50.) 50.

[9] E. Witten, Phys. Lett. B153 (1982)

[10] B.R. Greene, A. Shapere, C. Vafa and S.-T. Yau, Nucl. Phys. B337 (1990) 1.

[11] S. Ceccoti, Phys. Lett. B244 (1990) 23.

[12] C. Gundlach and M.E. Ortiz, Phys. Rev. D42 (1990) 2521.

[13] E. Witten, Phys. Rev. D44 (1991) 314.

[14] G.T. Horowitz and A. Strominger, Nucl. Phys. B350 (1991) 197.

[15] J.H. Horne and G.T. Horowitz, Nucl. Phys. B368 (1992) 444.

[16] E. Raiten, Fermilab preprint FERMILAB-PUB-91-338-T, Dec. 1991.

[17] P. Horava, Phys. Lett. B278 (1992) 101; D. Gershon, Tel Aviv University preprint TAUP-1937-91, Dec 1991.

[18] S. K. Kar, S. P. Khastgir and G. Sengupta, IP Bhubaneswar preprint IP/BBSR/9235, May 1992; S. Mahapatra, Tata Institute preprint 92-28, May, 1992.

[19] I. Bars and K. Sfetsos, USC preprint USC-92/HEP-B1, May 1992; I. Bars and K. Sfetsos, USC preprint USC-92/HEP-B2, May 1992; K. Sfetsos, USC preprint USC92/HEP-S1, June 1992; 
[20] A. Dabholkar and J. Harvey, Phys. Rev. Lett 63 (1989) 719; A. Dabholkar, G. Gibbonws, J. Harvey and F. Ruiz, Nucl. Phys. 340 (1990) 33; Soo Jong Rey, Phys. Rev. D43 (1991) 526.

[21] B.A. Campbell, M.J. Duncan, N. Kaloper and K.A. Olive, Nucl. Phys. B351 (1991) 778.

[22] J. Antoniades, C. Bachas, J. Ellis and D. Nanopoulos, Phys. Lett. B221 (1988) 393;

J. Antoniades, C. Bachas and A. Sagnotti, Phys. Let. B235 (1990) 255.

[23] A. Shapere, S. Trivedi and F. Wilczek, IAS preprint IASSNS-HEP-91/93, June 1991.

[24] A. A. Tseytlin, Cambridge University preprint, DAMTP-92-06, June 92 